\title{
Antibodies as mediators of brain pathology
}

Lior Brimberg ${ }^{1}$, Simone Mader ${ }^{1}$, Yuichiro Fujieda ${ }^{1}$, Yoshiyuki Arinuma ${ }^{1}$, Czeslawa Kowal ${ }^{1}$, Bruce Volpe $^{1}$ and Betty Diamond ${ }^{1 *}$

${ }^{1}$ Feinstein Institute for Medical Research, Manhasset, NY

*Corresponding author. Diamond, B. (BDiamond@NSHS.edu)

\begin{abstract}
The brain is normally sequestered from antibody exposure by the blood brain barrier. However, antibodies can access the brain during fetal development before the barrier achieves full integrity, and in disease states when barrier integrity is compromised. Recent studies suggest that antibodies contribute to brain pathology associated with autoimmune diseases such as systemic lupus erythematosus and neuromyelitis optica, and can lead to transient or permanent behavioral or cognitive abnormalities. We review these findings here, and examine the circumstances associated with antibody entry into the brain, the routes of access and the mechanisms that then effect pathology. Understanding these processes and the nature and specificity of neuronal autoantibodies may reveal therapeutic strategies towards alleviating or preventing the neurological pathologies and behavioral abnormalities associated with autoimmune disease.
\end{abstract}

(C) 2015. This manuscript version is made available under the Elsevier user license http://www.elsevier.com/open-access/userlicense/1.0/ 


\section{Introduction}

There has been much recent study of genetic variants that contribute to abnormal cognition or behavior. While this has been a fruitful area of investigation, there is growing evidence that environmental triggers, perhaps in conjunction with genetic risk factors, are also important in both early and late onset disturbances in brain function ${ }^{1}$. In particular, perturbations in both the innate and the adaptive immune system can alter brain development in the fetus as well as brain function in the adult ${ }^{2,3}$. Two major classes of immune effector molecules - cytokines and antibodies - have been demonstrated to affect brain development and brain function ${ }^{3-5}$. In particular, our appreciation of the spectrum of antibodies with this potential keeps growing. It is now appreciated that brain-reactive antibodies can arise as a result of autoimmune disease or as an untoward consequence of an anti-microbial response. Autoimmune diseases such as systemic lupus erythematosus (SLE) and neuromyelitis optica (NMO) are characterized by brain-reactive serology, and Sydenham's chorea, which develops after exposure to group A streptococcus, is characterized by so called signaling autoantibodies - anti-streptococcal antibodies that are cross-reactive with dopamine receptors ${ }^{6,7}$. Brain-reactive antibodies may also be a feature of paraneoplastic syndromes, arising through cross-reactivity with tumor antigens.

Here, we review the current understanding of the impact of antibodies on brain development and function. We examine the settings in which antibodies are able to access the 'immune privileged' environment of the central nervous system (CNS), and the routes used for this access. In the context of autoimmune disease, we discuss the known neuronal targets of antibodies, and the antibody-mediated effector mechanisms that mediate brain pathology. We center this discussion on two autoimmune diseases - SLE and NMO - presenting these as paradigms for the study of the potential contribution of antibodies to congenital and acquired brain disease. A deeper understanding of the nature and specificity of neuronal autoantibodies, 
and the circumstances and ways in which these antibodies access the CNS should enable new therapeutic strategies towards alleviating or preventing the neurological pathologies and behavioral abnormalities associated with autoimmune disease.

\section{The Blood Brain Barrier}

The blood-brain barrier (BBB) is the major interface between molecules in the circulation and the brain. Its architecture has recently been described as a two-walled moat surrounding the brain ${ }^{8,9}$ which separates blood from interstitial fluid. It is composed of endothelial cells tightly linked by specialized proteins that form the tight junction. Astrocytes lay down a basement membrane (glia limitans) in which pericytes reside. The endothelial barrier and the glia limitans help control the composition of the interstitial fluid in the brain and help shield the brain from the surrounding interstitial fluid. Barrier properties are most restrictive in the capillaries, and are less so in the venules. Perivascular macrophages sample the CSF in the space between the astrocytic and endothelial basement membrane, within postcapillary venules. They harbor phagocytic properties and might have important implications as antigen presenting cells.

The blood CSF barrier (BCSFB) separates blood from the CSF and is formed by epithelial cells of the choroid plexus, which possess unique apical tight junctions. In addition to serving a barrier function, the choroid plexus epithelial cells secrete CSF. This architecture permits a continuous interchange of CSF and interstitial fluid and has been recently named the glymphatic system ${ }^{10}$. The finding of functional lymphatic vessels, which connect the CSF with the deep cervical lymph nodes, allow passage of immune cells and immune molecules into the CNS. ${ }^{11,12}$.

The BBB begins to be formed early in embryonic development, following neovascularization of neural tube at embryonic day 10.5 (E10.5) ${ }^{13}$. There are many studies that have investigated the timing of BBB development during embryogenesis ${ }^{14}$. It is clear that even during the very early 
stages of brain development, there is a limitation on transit of molecules from the blood to brain parenchyma that is more restrictive than in other tissues. Our own studies, however, demonstrate that, in mouse, the BBB is not fully impenetrable to $\lg G$ until $~ E 17.5$ when there is almost total exclusion of IgG in the brain ${ }^{15}$. Presumably a similar window of vulnerability to $\lg G$ exists for the developing human brain ${ }^{16}$. Thus, there is a short window during the second trimester of gestation when maternal IgG crosses the placenta to enter the fetal blood stream and may access brain parenchyma. After this brief developmental window, the barrier prohibits $\lg \mathrm{G}$ access to the brain terminates. We have demonstrated that aspects of maternal physiology can impair the development of a competent BBB in the fetus. For example, female mice lacking gut microbiota harbor fetuses that never develop a proper BBB, and even as adults, the offspring have leaky barrier properties. Reconstitution of the adult offspring with a gut microbiome leads to the proper maturation of the BBB ${ }^{15}$. The spectrum of maternal abnormalities that cause either a transient or permanent deficiency in BBB integrity is not known.

The BBB is a dynamic structure and contact with various molecules can alter barrier integrity. Mechanisms for altering the barrier function include altering the integrity of the tight junctions between the endothelial cells, or affecting the capacity of the endothelial cells to internalize molecules through receptor mediated internalization, for example, in the transport of cytokines or chemokines ${ }^{17}$. Interestingly, many molecules that impair barrier integrity are soluble mediators released during inflammation. In sepsis, characterized by a dramatic activation of an innate immune response, it is well documented that there is a massive breakdown in barrier integrity ${ }^{18}$. Because imaging studies of the BBB are still relatively insensitive to small changes in barrier function, we do not know if chronic inflammatory diseases characterized by a lower level of inflammatory mediators in the plasma might be associated with a low-level, but chronic, barrier breach that might have clinical consequences. It is well documented that barrier 
modulators include bacterial and viral infection, systemic inflammation, trauma, brain ischemia, and stress, as well as specific agonists of endothelial receptors like nicotine, caffeine and cocaine ${ }^{19}$. The exact mechanisms by which each of these conditions alters barrier integrity are not known, although many would appear to alter the integrity of the tight junctions. Two additional aspects of BBB function need to be mentioned. First, insults causing inflammation within the brain, with activation of inflammatory properties of microglial cells, can also impair barrier integrity. Second, the microvascular endothelial cells of the brain are specifically designed to shield the brain from IgG and have transport receptors for immunoglobulin, the neonatal FCR (FcRn), usually located at the luminal surface of the endothelial cell and responsible for transporting IgG into tissue, at the abluminal surface where it functions to transport IgG out of the perivascular space and interstitial fluid. Under healthy conditions, IgG is removed from the central nervous system (CNS) by reverse transcytosis across the BBB ${ }^{20}$, mediated by the FcRn ${ }^{21}$. When BBB is compromised, net influx of IgG is increased such that FcRn are saturated, resulting in accumulation of $\lg G$ in the parenchyma (Figure 1).

There are also reports of impaired barrier integrity with aging, Alzheimer's disease and atherosclerosis ${ }^{22}$. ApoE deficient mice, an important model of both atherosclerosis and Alzheimer's disease, exhibit a BBB that is leaky to small molecules and in some cases to $\lg$ G. ${ }^{23-25}$. Given the growing list of conditions with altered BBB function, it is probable that we will continue to identify even more conditions associated with altered BBB properties. Whether antibody can enter the brain without traversing tight junctions is not established, although the mechanism of transcytosis has been exploited for therapeutic antibody delivery in model systems ${ }^{26}$.

In contrast, only a few mechanisms are known to maintain BBB integrity. Glucocorticoids appear to support barrier properties ${ }^{27}$, as do interferons ${ }^{28-30}$. Type 1 interferon prevents barrier 
disruption through multiple mechanisms. It reduces neutrophil invasion following inflammatory insults and diminishes their production of matrix metalloproteinase 9 (MMP9) ${ }^{31}$. It also increases expression of ecto-5'-nucleotidase (CD73) on endothelial cells, thereby increasing the local concentration of adenosine, an anti-inflammatory molecule ${ }^{32}$. Indeed, the therapeutic efficacy of $\beta$ interferon in multiple sclerosis may, in part, reflect its effects on barrier integrity ${ }^{33}$.

Studies in rodent models have demonstrated that the BBB is not a homogeneous structure and that receptors for modulators of barrier integrity are present at different density on the lumen of endothelial cells in different regions of the brain. For example, we have demonstrated that systemic administration of bacterial lipopolysaccharide (LPS) will permit IgG to access primarily the hippocampus ${ }^{34,35}$ while systemic administration of epinephrine will expose cells mainly in the amygdala to blood-borne substances ${ }^{36}$. It is possible that the regional distribution of TLR4 and TLR5 may mediate the specific hippocampus impairment by LPS ${ }^{37}$, while epinephrine might cause selective leak of the BBB by increasing differentially the cerebral blood flow in the amygdala ${ }^{38}$ and therefore induce changes in vascular permeability ${ }^{39}$. Insufficient information currently exists of the regional distribution of TNF receptors, complement component receptors, and more that modulate BBB integrity. The regional specificity of BBB compromise allows the same circulating antibody to have different effects on brain function depending on the region of exposure to antibody. In addition, certain areas of the brain, the circumventricular organs, lack an intact BBB, and thus might be important regions for antibody passage ${ }^{40}$. Antibodies may also alter brain function if they are produced locally within the CNS or the meninges, by infiltrating B cells. In MS, where oligoclonal IgG bands in the CSF are a hallmark of the disease ${ }^{41}$. B cells have been identified in tertiary lymphoid structures of the meninges and Virchow Robin spaces, where antigen specific B cells clonally expand ${ }^{42}$. Understanding whether brain-reactive antibodies are produced by B cells sequestered in the brain or by 
peripheral B cells has important therapeutic implications, however, most brain pathology caused by antibody appears to reflect transit of IgG into the brain rather than local production.

\section{Antibodies in the adult brain}

Over the last decade, several antibodies have been described which bind to different extracellular or intracellular central nervous system (CNS) antigens (Table 1; Key Table). For example, a substantial number of patients with neoplasms that trigger production of antibodies to oncofetal proteins and elicit neuro-muscular symptoms also suffer from cognitive impairment associated with antibodies that bind to neuronal antigens (for review see ${ }^{3}$ ). In many of these patients, the development of antibodies is secondary to the presence of a primary tumor that expresses neuronal antigens. Antibodies that bind brain antigen are also present in patients with autoimmune disease, including celiac disease, type 1 diabetes and thyroiditis ${ }^{43-45}$. Many of these appear to bind antigens that are not restricted to the brain or to bind antigens in other organs and cross-react with brain antigens. In many patients, there is no discernible brain pathology, but more and more there is awareness that these diseases may be associated with cognitive impairment. Whether, and to what degree, antibodies are responsible for the neurocognitive symptoms requires further study.

For the present discussion we focus on SLE and NMO, two autoimmune diseases that have been shown to be B cell-dependent ${ }^{46,47}$. Neuropsychiatric SLE (NPSLE) is characterized by autoantibodies directed to neuronal antigens in a least a substantial group of patients. These include anti-N-methyl-D-aspartate (NMDA) receptor (NMDAR) antibodies, which are a crossreactive subset of anti-DNA antibodies, the hallmark autospecificity in SLE, and anti-ribosomal P

protein (anti-P) antibodies. Both these antibody specificities can induce NPSLE in mice ${ }^{35,36,48}$. In contrast, as we will discuss further in later sections, in NMO, antibodies are directed to antigen on glial cells. 


\section{Autoantibodies in SLE and the induction of behavioral abnormalities}

\section{Anti-DNA/NMDAR antibodies}

SLE patients are known to harbor autoantibodies of multiple specificities, such as antiphospholipid, anti-Ro and anti DNA antibodies. Anti DNA antibodies are the most frequent autospecificity in SLE ${ }^{49}$. A subset of anti-dsDNA autoantibodies has been demonstrated to cross-react with NMDAR (anti-DNA/NMDAR antibody) ${ }^{50}$. The NMDAR is a glutamate receptor and ion channel protein found in nerve cells. Glutamate is a major excitatory neurotransmitter in brain involved in synaptic plasticity and memory function. The anti-DNA/NMDAR antibodies bind to GluN2A and GluN2B subunits of the NMDAR, which contain the consensus peptide sequence D/E-W-D/E-Y-S/G (DWEYS) ${ }^{51}$. Both murine and human anti-DNA/NMDAR antibodies, the latter cloned from B cells of SLE patients, cause dose-dependent excitotoxic neuronal death when injected directly into the hippocampus ${ }^{50,52}$ of a non-autoimmune, unmanipulated mouse. The anti-DNA/NMDAR antibodies preferentially bind the active receptor, presumably prolonging the time when the channel is open to calcium influx ${ }^{52}$. Overstimulation of NMDAR leads to high influx of calcium into the cell, causing neuronal dysfunction and cell death.

BALB/c mice immunized with the DWEYS peptide in a multimeric configuration (MAP-DWEYS) produce anti-DNA/NMDAR antibodies ${ }^{50}$. Despite the presence of anti-DNA/NMDAR antibodies in the serum of these mice, there is no detectable binding to neurons or damage in neurons of the brain that could be detected in histologic analyses. In this model, a breach in the integrity of the BBB is required for antibody to access brain tissue and affect neuronal function and viability. LPS injection as a surrogate for infection leads to the extravasation of the antibody into the hippocampus and, consequently, focal neuronal loss and cognitive deficits ${ }^{35}$. In contrast, epinephrine injection in mice as a surrogate for stress allows antibody to penetrate the 
amygdala, and neuronal loss in that region leads to emotional disturbances that can be assessed in studies of the response to aversive stimuli ${ }^{36}$. These data show that regional disruption of the BBB is dependent on the agent used to modify the BBB and, thus, that the same antibody can cause variable behavioral changes.

Two weeks post-BBB breach, FDG-PET imaging of MAP-DWEYS immunized mice revealed a lowered glucose uptake, a surrogate for metabolic activity, in the hippocampus or amygdala respectively, as compared to the baseline signal, whereas control mice, immunized with MAP only and given LPS, showed heightened glucose uptake ${ }^{53}$. At 4 weeks post-BBB breach, both groups exhibited increasing glucose uptake in the affected region. In control mice, glucose uptake was directly related to neuron number, while in mice with anti-DNA/NMDAR antibodies, there was an inverse relationship between neuron number and metabolism. The increase in glucose uptake that occurs between 2 and 4 week post-BBB breach in the mice harboring the lupus-like antibodies is thought to reflect enhanced synaptic and/or glial activation. Importantly, hippocampal increase glucose uptake in the patients is commonly seen in SLE ${ }^{54}$ subjected to FDG-PET imaging, suggesting that they might also have sustained glial activation.

Most recently, we have demonstrated that there are late sequelae of anti-DNA/NMDAR antibody penetration of the hippocampus ${ }^{55}$. After antibody can no longer be detected in brain tissue, neurons in the hippocampus undergo a loss of dendritic processes. Hippocampal neurons possess place cell activity and are responsible for generating spatial maps of the environment. Each neuron fires when the mouse is in a particular location (place field). Two-4 weeks after antibody penetrates the hippocampus and cues the acute death of some neurons, surviving neurons display decreased dendritic complexity and enlarged place field size along with diminished spatial memory. We speculate that the alteration in dendritic processes may also reflect glial activation ${ }^{55}$. 
Many reports have indicated an association of autoantibodies with mechanism of psychiatric manifestations in human SLE patients. In some studies, anti-DNA/NMDAR antibodies are elevated in serum from patients with active non focal NPSLE ${ }^{56,57}$, such as confusional state or psychosis. However, in other studies the presence of these antibodies in serum was not associated with active diffuse NPSLE ${ }^{58-63}$. These results suggest that when anti-DNA/NMDAR antibodies are present only in serum, they do not mediate neuropsychiatric manifestations. However, the level of anti-DNA/NMDAR antibodies in the cerebrospinal fluid (CSF) was significantly elevated in patients with active non focal NPSLE, as compared to NPSLE patients with focal NPSLE or to patients with non-inflammatory disease in the CNS ${ }^{63}$. These results suggest that the direct access of pathogenic autoantibodies to the CNS is a key factor for the development of neuropsychiatric manifestations in SLE. In SLE, antibodies appear to access the brain secondary to BBB compromise, which can be assessed by the presence of albumin in the CSF ${ }^{64}$. There is little evidence for infiltration of antigen-specific B cells into brain parenchyma.

Interestingly, anti-DNA/NMDAR antibodies have been reported to react with human umbilical vein endothelial cells, resulting in the production of inflammatory cytokines ${ }^{65}$. Thus, antiDNA/NMDAR antibodies might directly damage brain vessels, and contribute to the breakdown of the BBB ${ }^{6}$.

\section{Anti-ribosomal $P$ antibodies}

Anti-P antibodies were first identified by the binding to $\mathrm{C}$-terminal regions of three ribosomal $\mathrm{P}$ proteins ( $\mathrm{P} 1, \mathrm{P} 2$ and $\mathrm{P} 0){ }^{67}$. The binding site on the ribosomal proteins has been characterized and termed P epitope. Recently these antibodies were shown to cross-react with a high molecular weight integral plasma membrane protein of unknown function, called neuronal 
surface $P$ antigen (NSPA), which possesses a $P$ epitope at the cell surface ${ }^{66}$ and is expressed exclusively in neurons. Passive transfer experiments in mice showed that anti-P antibodies isolated from SLE patients induced smell alterations ${ }^{68}$, depression-like manifestations ${ }^{69}$ and memory impairment ${ }^{48}$. Immunohistochemistry analyses of NSPA expression patterns in the brain revealed that NSPA is present in multiple areas, including the cortex, amygdala, and hippocampus and is expressed exclusively in neurons. ${ }^{70}$. When added to primary neuron cultures, anti-P antibody induces calcium influx followed by apoptosis, and similarly, direct injection of anti-P antibodies into the brain of normal mice results in neuronal death through an excitatory glutamatergic pathway ${ }^{66}$.

Anti-DNA/NMDAR antibodies and anti-P antibodies both recognize neuronal cell surface antigens that enhance glutamate induced neuronal activation, but they act through different mechanisms. At low concentrations, anti-DNA/NMDAR antibody increases excitatory postsynaptic potentials (EPSPs) by directly interacting with an epitope of NMDAR that becomes accessible in the open channel configuration once the $\mathrm{Mg}^{2+}$ plug has been removed. At higher concentrations, an excess of calcium flux into the cell leads to neuronal death ${ }^{52}$. In contrast, anti-P antibody enhances glutamatergic transmission in the presence of $\mathrm{Mg}^{2+}$ through a mechanism mediated by NSPA and involving a-amino-3-hydroxy-5-methyl-4-isoxazolepropionic acid (AMPA) receptor and NMDAR activation ${ }^{70}$. Like anti-DNA/NMDAR antibodies, intravenously injected anti-P antibodies impaired memory function in mice alter LPS administration ${ }^{70}$. Anti-P antibodies can potentiate the pathogenic effects of anti-DNA/NMDAR, promoting the active configuration of the NMDAR, and the concomitant presence of both antibodies might constitute another factor in brain dysfunction variability.

Anti-P antibodies are major autoantibodies that are present in serum of $40-50 \%$ of active nonfocal NPSLE patients ${ }^{71,72}$. In vitro, anti-P antibodies from patients with diffuse NPSLE can 
directly react with the surface of human peripheral blood monocytes, which results in TNF- $\alpha$ production ${ }^{73}$. Therefore, anti-P antibodies could be associated with autoantibody-mediating inflammation in the CNS through the mechanism of BBB rupture by monocyte-derived TNF- $\alpha$ with or without infiltration of activated macrophage into CNS ${ }^{73}$. Accordingly, anti-P antibodies might also activate the immune system, access the CNS, and directly damage neurons in SLE patients ${ }^{48,73}$.

Taken together, these findings suggest that two phases are required for the development of neuro- psychiatric or neurocognitive manifestations in human lupus patients. The first phase involves the direct access of pathogenic autoantibodies to the CNS, mainly as a consequence of influx from the systemic circulation to the CNS following the breakdown of BBB. The second phase involves either direct or microglia-mediated damage of neurons by autoantibodies in the CNS. It should also be noted that whereas anti-DNA/NMDAR and anti-ribosomal P antibody specificities have been studied extensively, other brain-reactive antibodies have been reported in SLE ${ }^{74}$. Furthermore, there is a growing awareness that antibodies implicated in other autoimmune diseases may cross-react with brain targets. For example, anti-gliadin antibodies present in patients with celiac disease have been shown to cross-react with antigens expressed by purkinje cells in the cerebellum ${ }^{75}$, and to bind to neuronal synapsin I ${ }^{44}$. Thus, the paradigm described above for anti DNA/NMDAR and anti-P antibodies may hold true for antibodyassociated neuropsychiatric and neurocognitive impairment in many autoimmune diseases.

\section{Neuromyelitis Optica (NMO) - cognitive impairments resulting from antibodies targeting non-neuronal cells}

Brain-reactive antibodies that bind to non-neuronal cells can also alter neuronal viability or function through mechanisms that are not yet well described. Antibodies that result in glial cell death can impair neuronal survival. Patients afflicted with the autoimmune disease NMO have 
antibodies directed to astrocytes in their serum ${ }^{76}$. Lennon and colleagues ${ }^{77}$ discovered that those astrocyte antibodies in patients with NMO bind the antigen aquaporin-4 (AQP4), a water channel protein that is expressed on astrocytic foot processes surrounding blood vessels and that controls the flow of water in and out of the brain. A pathogenic role of AQP4 antibodies was first suggested due to pathological findings of a massive deposition of antibody and complement components surrounding blood vessels ${ }^{78}$. The mechanism through which the loss of astrocytes leads to demyelination and neuronal loss is not fully understood; however, there is emerging evidence that astrocytes can regulate myelination ${ }^{79}$, maybe by modulating the function of oligodendrocytes. Alternatively, ex vivo data suggest that patients' antibodies which target astrocytes can damage oligodendrocytes secondarily possibly due to a bystander effect caused by glutamate-mediated excitotoxicity ${ }^{80}$.

Serum AQP4 antibodies can help to diagnose the disease at an early stage, and thereby can enable early treatment. This is particularly important as NMO can closely resemble other diseases such as Multiple sclerosis (MS), which often require different treatment approaches ${ }^{81}$. Confirming the importance of autoantibodies in NMO pathology, antibody or B cell depleting treatment are beneficial for NMO patients, whereas interferon $\beta$, commonly used for treating MS, can exacerbate the disease ${ }^{81}$. Clinical assays for detection of AQP4-IgG have high sensitivity and specificity, and thus antibody serology is included as a diagnostic criterion for NMO ${ }^{82,83}$. Approximately $70-90 \%$ of $\mathrm{NMO}$ patients are seropositive for AQP4-IgG ${ }^{84,85}$, and this range is thought to depend on differences in the detection assays. AQP4-lgG recognizes conformational epitopes and therefore, antibodies can be detected with highest sensitivity and specificity using cell-based assays using a transfected cell line expressing AQP4 on their surface ${ }^{86-88}$. NMO was initially described as a disease which selectively targets astrocytes in the optic nerve and spinal cord and spares the brain ${ }^{89}$; however, brain MRI abnormalities are very common in patients with AQP4-lgG ${ }^{90}$ and brain lesions are found in areas of high AQP4 expression, most 
prominently in the grey matter. Indeed, NMO patients exhibit cognitive impairment and cortical neuronal loss ${ }^{91}$.

Intravenous injection of AQP4-lgG to rodents, without disrupting the BBB, does not result in a neuropsychiatric phenotype ${ }^{47}$. This finding is consistent with the observation that AQP4 IgG can be detected in the serum of NMO patients many years prior to the full onset of disease ${ }^{92}$. However, the inflammatory setting that leads to the breach of the BBB in NMO is not well understood. It is also not clear how the antibodies exert their pathologic effect. Some studies have suggested that AQP4 antibodies damage the brain in concert with pathogenic T cells; others studies suggest that complement or antibody dependent cellular cytotoxicity are the critical mechanisms ${ }^{93-95}$.

In vivo models of NMO either require induction of experimental autoimmune encephalitis (EAE) to activate BBB disrupting pathogenic T cells reactive to antigens expressed in the brain ${ }^{47,96}$, or direct intracerebral injection of antibodies together with human complement to mediate CNS injury ${ }^{97}$. It is possible that there is not a single mechanism of BBB insult in NMO. Different mechanisms of BBB compromise might be specific to distinct regions of brain or spinal cord and thus might correlate with the heterogeneous pattern of lesions that have been described in NMO 98. Moreover, some mechanisms might permit only lgG to enter the brain as we have seen in our LPS-induced model of BBB compromise while others such as EAE induction permit cellular entry and in situ production of $\lg { }^{99,100}$. Evidence, albeit rare, supporting the latter model comes from the identification of transient oligoclonal $\lg G$ bands in CSF of some patients ${ }^{93}$, suggesting that the antibodies in the CSF may be made from B cells residing in brain parenchyma where they might locally produce antibodies, as in MS.

In vivo models differ in terms of where the breach of the BBB occurs, and the time it takes for this to happen. For example, the choice of the antigen specific T cells for in vivo NMO/EAE 
models results in different regional brain lesions and varying disease severity ${ }^{101}$. Whether specific brain-reactive T cells, perhaps AQP4 reactive T cells ${ }^{94,102,103}$, are necessary to initiate or propagate the disease remains unresolved ${ }^{94,102}$. However, T cells are present in brain lesions of $\mathrm{NMO}$ patients ${ }^{101}$. A role for cytokines has been demonstrated by injecting cytokines directly into the striatum of AQP4 seropositive rats ${ }^{104}$. Interleukin-1 beta (IL1b) was the only cytokine capable of inducing NMO lesions outside the needle track. Interestingly, IL-1b is also found in active human NMO lesions ${ }^{104}$. These rodent NMO models partially mimic human disease, specifically the initial stages of the disease ${ }^{105}$.

Overall, these studies demonstrate that antibodies capable of causing brain pathology can remain in the circulation for long period of time. So long as they do not penetrate the $\mathrm{BBB}$, there are no CNS sequelae. Mechanisms of BBB breach in the adult organism can permit only soluble molecules to penetrate brain parenchyma or cells as well as soluble molecules. The mechanism, and the location, of BBB breach dependent on the particular stressor. Once antibodies penetrate the CNS, they may alter brain function through damaging cells, either neurons or glial cells, or through altering cell function. Finally there are both immediate and late sequelae of antibody exposure. The availability of appropriate animal models is critical as studies of patients may be confounded by cohorts that express similar antibodies but differ with respect to mechanism of barrier breach and state of disease.

\section{Maternal antibodies and the fetal brain}

During pregnancy, the placenta provides a barrier between maternal and fetal circulation while simultaneously allowing the transfer of a wide range of substances to the fetus, including nutrients. Among these factors are maternal lgG molecules, which start to cross the placenta around the second trimester and provide the developing fetus with humoral immunity, since the fetal immune system is immature at this stage (for a review see ${ }^{106}$ ). Nevertheless, there are 
circumstances in which placental transmission of antibodies is deleterious to the infant. For example, neonatal lupus is a rare disorder in which maternal anti Ro/SSA and/or La/SSB antibodies can lead to transient symptoms including skin rash, liver problems, or low blood cell counts, which typically disappear after six months with no lasting effects ${ }^{107}$. These antibodies can lead also to congenital heart block with a permanent structural heart defect ${ }^{107}$. Transient and/or permanent tissue injury secondary to maternal antibodies has also been reported in myasthenia gravis ${ }^{108}$, anti-phospholipid syndrome and in other autoimmune diseases ${ }^{109}$.

Once maternal lgG crosses the placenta, the antibodies can penetrate all tissues including the developing brain. Importantly, although the mature BBB prevents IgG entry into the adult CNS, in the fetus $\lg$ can penetrate the brain ${ }^{15}$, potentially through an immature BBB, through the blood-CSF barrier, or perhaps through the vascular fenestration in the circumventricular organ. In mice, we have recently confirmed that the brain is accessible to maternal antibodies from embryonic day (E) 13.5 to E16.5 ${ }^{15}$. Maternal autoantibodies in patients afflicted with SLE and NMO have been shown to affect fetal neurodevelopment, as we discuss below. In addition, we discuss the evidence for a role for maternal antibodies in autism spectrum disorder (ASD).

\section{Maternal autoantibodies in SLE}

Several studies have suggested that children, particularly the sons of mothers with SLE, but not fathers, are at increased risk for learning disabilities ${ }^{110-113}$. Other epidemiological studies suggested that children born to mothers with SLE have, in general, a higher risk to neurodevelopmental disorders including ASD ${ }^{114}$. Studies in mice have further sustained a potential link between exposure to SLE and neurodevelopmental disorders. We showed cortical abnormalities in fetuses exposed in utero to monoclonal anti DNA/NMDAR IgG, cloned from an SLE patient ${ }^{115}$. Moreover, we studied a mouse model in which dams harbor anti-DNA NMDAR specific autoantibodies throughout gestation to demonstrate that fetal brain exposed to high 
titers of anti DNA/NMDAR antibodies showed increased neuronal cell death, abnormal neuronal proliferation and thinning of the cortical plate. Live offspring in these studies showed delayed reflexes as neonates and cognitive impairments in cortical dependent tasks as adults ${ }^{115}$. Interestingly, these developmental effects were evident primarily in male offspring as exposure in utero to high titers of anti DNA/NMDAR antibodies led to a preferential loss of female fetuses, apparently due to the apoptosis effects mediated by these antibodies on neurons expressing GluN2A in the midbrain. GluN2A is expressed earlier in female compared to male midbrain and mice lacking GluN2A exhibited no fetal loss in this model ${ }^{116}$. Interestingly, it has been recently confirmed that mothers with SLE are more likely to have a male child ${ }^{117}$. This finding further supports the hypothesis that maternal antibodies may have gender-specific effects on brain development.

It should be mentioned that additional maternal anti NMDAR antibodies against GluN1 have been described as leading to cortical dysplasia in the offspring in NMDA encephalitis ${ }^{118}$.

\section{Autism Spectrum Disorder (ASD)}

The possibility that maternal autoantibodies can lead to a subset of ASD has been entertained for more than a decade. Several investigators have identified the presence of antibodies that bind to human fetal brain tissue in a subset of women who have children with ASD ${ }^{119-122}$. In the largest study that has been performed so far including 2789 mothers of an ASD child, we have shown that brain-reactive antibodies are present at higher frequency in mothers of an ASD child. $10.5 \%$ of mothers of an ASD child harbor antibodies with strong reactivity to mouse brain antigens compared to $2.6 \%$ of unselected age-matched women ${ }^{119}$. When researchers gave these antibodies to pregnant mice and monkeys, they caused abnormal behavior in their offspring including increased motor activity, stereotypic behavior anxiety-like behavior and 
alterations in sociability ${ }^{123-128}$. A recent study showed prenatal exposure to anti-brain antibodies also alters neuronal proliferation, and brain size ${ }^{129}$.

Unlike the offspring of SLE mothers who have a pre-identified medical condition, in ASD the mothers themselves may have no obvious autoantibody-related pathology; however, brainreactive antibodies appear to be more common in women with autoimmune disease. Atladóttir and colleagues have shown that mother with rheumatoid arthritis or celiac disease have almost double increased risk to have a child with ASD ${ }^{130}$. Our own study associated the presence of maternal anti-brain antibodies with increased autoimmune disease in mothers of an ASD ${ }^{119}$.

The specificity of the brain-reactive antibodies associated with ASD is an area of active investigation ${ }^{120}$. Several neuronal antigens expressed in the developing brain have already been suggested as possible targets ${ }^{120}$. Given the heterogeneity of the disease, it is highly likely that different antibody specificities will be associated with distinct symptoms of ASD. For example, Braunschweig and colleagues have shown that maternal serum reactive with a particular molecular weight band on Western blots of brain correlated with lower expressive language scores in the affected offspring while reactivity to a different molecular weight band size correlated with increased irritability ${ }^{131}$.

\section{Maternal antibodies in NMO}

In NMO, a disease that affects primarily women of childbearing age, the possible effect of AQP4 antibodies on fetal CNS development has only been recently addressed. During pregnancy, women with AQP4 IgG have a more severe disease, develop preeclampsia more often and have an increased risk of miscarriages ${ }^{132,133}$. Case reports have suggested placental inflammation or birth defects in offspring that were exposed in utero to AQP4 $\operatorname{lgG}{ }^{132,134}$. Our own work, with pregnant mice injected intravenously with a single dose of AQP4 antibody 
isolated from a patient with active NMO, suggests a titer dependent effect of maternal AQP4 IgG on offspring (unpublished Mader \& Brimberg et al.). At high titers maternal AQP4 IgG impairs survival of offspring and at lower titers AQP4 IgG can affect the offspring's brain.

\section{Concluding Remarks}

Classical roles for antibodies are to assist in cellular debris removal, to eradicate harmful pathogens and to neutralize toxins. For the latter two functions especially, a diverse repertoire is required. Although it is clear that antibodies can be responsible for significant brain pathology, we have raised the hypothesis before ${ }^{3}$ that negative selection for brain antigens might be deleterious leaving the host with too narrow an antibody varsity and a diminished ability to protect effectively against infections and toxins. Indeed, it is notable that diversification of the B cell repertoire occurs after the BBB is formed, limiting the exposure of developing B cells to brain antigens. This hypothesis predicts that all individuals may develop brain-reactive antibodies as part of a protective immune response, and that the BBB serves two important functions. First, it sequesters brain antigens and limits their contribution to negative selection of B cells. Second, it protects the brain whenever anti-brain antibodies are made. We, therefore, speculate that anti-brain antibodies may be commonly present in healthy individuals. The purging of the immature B cell repertoire of autoreactive cells occurs in the bone marrow and spleen after the BBB has been formed. All mechanisms of B cell tolerance that we understand to date require that antigen to be present and trigger $\mathrm{B}$ cell receptor $(\mathrm{BCR})$-mediated tolerance mechanisms. It is possible therefore, that many brain-reactive $B$ cells mature to immunocompetence. Usually, these B cells may be of no consequence. Even if they are activated, possible through cross-reactivity with microbial antigens, as has been demonstrated for $\mathrm{B}$ cells cross-reactive with streptococcus and the dopamine receptor ${ }^{6,7}$, the antibodies are not able to bind to the brain antigen so long as the BBB retains it impermeability to antigen. If, 
however, the integrity of the BBB is compromised, these antibodies can contribute to alterations in brain function.

The increasing recognition of the potential damaging impact of brain-reactive antibodies on the developing fetal brain and the adult brain requires that we consider therapeutic strategies to protect the brain. Antibody-depletion strategies such as plasmapheresis are impractical as long-term therapy, B cell depletion or blockade of B cell activation is potentially immunosuppressive. A novel, potentially non-immunosuppressive, strategy is to protect or enhance BBB integrity. Most brain-reactive antibodies that cause pathology in adults appear to enter the brain primarily as soluble molecules; thus, more studies of the mechanisms that regulate the $\mathrm{BBB}$ are needed as well as more modalities for non-invasive or minimally-invasive assessments of BBB function. Protecting the BBB has the advantage that it does not require knowledge of all pathogenic antigenic specificities.

An alternative strategy might be the use of decoy antigen to neutralize serum antibodies such that they no longer bind to target tissue ${ }^{135}$. The requirements for this strategy approach are a lack of toxicity or immunogenicity. This approach has been pioneered in ex vivo studies, but has not yet been demonstrated to show efficacy in vivo ${ }^{135}$. Its advantage is that it is less likely to perturb other aspects of physiology, is non-immunosuppresive, and does not require the knowledge for each disease on how antibodies penetrate the BBB. It is our hypothesis that decoys antigens might be most useful in conditions of transient production of brain-reactive antibodies, such as during infection.

One important question that has not yet been rigorously addressed is whether all brains are equally vulnerable to antibody-mediated pathology or what makes some brains more vulnerable, either during fetal development or in adulthood. We would speculate that vulnerability may 
involve genetic factors as well as antibody exposure or the coexistence of other modulators or stressors of brain cell function or viability, respectively.

Finally, it is interesting to ask whether we might use antibodies to improve brain function. It may be that antibodies could enhance neurotransmitter signaling in conditions characterized by a deficiency in these processes. The same antibodies that have pathogenic effects in individuals with normal brain function might improve brain function in some individuals with perhaps diminished NMDAR or dopamine receptor function. This intriguing possibility awaits further investigation. 
Table 1: Antibodies mediate brain dysfunction

\begin{tabular}{|c|c|c|}
\hline Etiology & Disease & Defined Antigen \\
\hline \multirow{15}{*}{ Malignancy } & Hodgkin's lymphoma & $\begin{array}{l}\text { AMPAR, mGluR1, } \\
\text { mGluR2, and mGluR5, } \\
\text { Tr (PCA1) } 136\end{array}$ \\
\hline & SCLC & $\begin{array}{l}\text { AMPAR, mGluR1, } \\
\text { mGluR2, and mGluR5 } \\
\text { Amphiphysin/synaptic vesicle } \\
\text { GABA (B1), CV2/CRMP5, } \\
\text { Gephyrin, Hu (ANNA1), Ma, } \\
\text { Recoverin, Ri (ANNA2) 137-141 }\end{array}$ \\
\hline & non-SCLC & GAD (GAD65, GAD67) ${ }^{142}$ \\
\hline & Ovary dermoid & 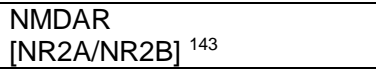 \\
\hline & Thymoma & $\begin{array}{l}\text { AMPAR, mGluR1, } \\
\text { mGluR2, and mGluR5 } \\
\text { GAD (GAD65, GAD67) } \\
\text { Caspr2, CV2/CRMP5, } \\
\text { Gephyrin 141,144 }\end{array}$ \\
\hline & Breast & $\begin{array}{l}\text { AMPAR, mGluR1, } \\
\text { mGluR2, and mGluR5 } \\
\text { Amphiphysin/synaptic } \\
\text { Vesicle, Hu (ANNA1), Ma, Ri } \\
\text { (ANNA2), Yo (PCA1), Zic4 } \\
\text { (PCA1) 141,145 }\end{array}$ \\
\hline & Thymic carcinoma & $\begin{array}{l}\text { AMPAR, mGluR1, } \\
\text { mGluR2, and mGluR5, Ri } \\
\text { (ANNA2) }{ }^{141,146}\end{array}$ \\
\hline & Endometrial carcinoma (rare) & Caspr2 144 \\
\hline & Teratoma & $\begin{array}{l}\text { NMDAR } \\
{\left[\text { NR2A/NR2B] }{ }^{141}\right.}\end{array}$ \\
\hline & Renal carcinoma & CV2/CRMP5 \\
\hline & pancreatic carcinoma & $\begin{array}{l}\text { GAD (GAD65, GAD67) } \\
\text { Gephyrin } 142\end{array}$ \\
\hline & Prostate & Hu (ANNA1) ${ }^{147}$ \\
\hline & Ovary & $\begin{array}{l}\text { Hu (ANNA1), Ri (ANNA2), Yo } \\
\text { (PCA1), Zic4 (PCA1) } 148\end{array}$ \\
\hline & Bladder & Hu (ANNA1) ${ }^{149}$ \\
\hline & Germ cell (testis) & $\mathrm{Ma} 150$ \\
\hline Infection & $\begin{array}{l}\text { Post-Streptococcal Movement } \\
\text { Disorders Sydenham's } \\
\text { chorea\& PANDAS }\end{array}$ & $\begin{array}{l}\text { lysoganglioside } \\
\text { Dopamine D1 and D2 } \\
\text { receptors } 6,7,151\end{array}$ \\
\hline \multirow{8}{*}{$\begin{array}{c}\text { Autoimmunity } \\
\text { (Antibodies are known } \\
\text { to cause disease) }\end{array}$} & \multirow[t]{2}{*}{ Neuropsychiatric SLE } & NR2A/NR2B ${ }^{50}$ \\
\hline & & Neuronal surface $\mathrm{P}$ antigen ${ }^{70}$ \\
\hline & Neuromyelitis Optica & AQP4 ${ }^{76,77}$ \\
\hline & ADEM & MOG $^{152}$ \\
\hline & \multirow[b]{2}{*}{ Limbic Encephalitis } & AMPAR (GluR1, GluR2) ${ }^{141}$ \\
\hline & & $\begin{array}{l}\text { NMDAR } \\
\text { NR1/NR2B }{ }^{153,154}\end{array}$ \\
\hline & Rasmussen Encephalitis & GluR3 $^{155}$ \\
\hline & Stiff Person Syndrome & $\begin{array}{l}\text { GAD, Gephryin GABA(A), } \\
\text { GABA (B1) }\end{array}$ \\
\hline
\end{tabular}




\begin{tabular}{|c|c|c|}
\hline & & Anti-Amphiphysin ${ }^{157}$ \\
\hline & & Synapsin $1^{44}$ \\
\hline & Cenlac Disease & Transglutaminase ${ }^{158}$ \\
\hline Autoimmunity & Limbic Encephalitis & $\operatorname{Lgi1/Caspr2}^{144,159,160}$ \\
\hline (Antibodies are NOT & Hashimoto's Encephalitis & $\begin{array}{l}\text { aldehyde reductase, } \\
\text { thyroglobulin } 45,161\end{array}$ \\
\hline $\begin{array}{l}\text { known to cause } \\
\text { disease) }\end{array}$ & Autism & $\begin{array}{l}\text { LDH, Cypsin,STIP-1, CRMP1, } \\
\text { CRMP2, YB-1 }{ }^{120}\end{array}$ \\
\hline
\end{tabular}

Abbreviations: AMPAR, $\alpha$-amino-3-hydroxy-5-methyl-4-isoxazolepropionic acid receptor; mGluR, metabotropic glutamate receptor; AQP4, astrocytic aquaporin-4 water channels; GABA, $y$-aminobutyric acid; GAD, glutamic acid decarboxylase; Caspr2,contactin-associated protein-like 2; CRMP5, collapsin response mediator protein 5; CRMP1/CRMP2, collapsin response mediator protein 1/2; Lactate dehydrogenase; LDH, Lgi1, leucine-rich, glioma-inactivated 1; NMDAR, N-methyl-D-aspartate receptor; SCLC, small-cell lung cancer; NR1, NR2A, and NR2B, subunits of the NMDAR; MOG; myelin oligodendrocyte glycoprotein; PANDAS, pediatric autoimmune neuropsychiatric disorders associated with streptococcal infections; STIP-1, stress-induced phosphoprotein 1; YB-1, Y-Box-Binding protein 1.

\section{Acknowledgments}

The authors thank Benjamin Obholzer for illustration. The research was funded by the NIAID, Department of Defense AR130137, and The Simons Foundation. LB is a recipient of a Brain and Behavior NARSAD Young Investigator Foundation Grant. SM is a recipient of SLE lupus foundation fellowship.

\section{References}

1. Mazina, V., et al. Epigenetics of autism-related impairment: copy number variation and maternal infection. J Dev Behav Pediatr 36, 61-67 (2015).

2. Rivest, S. Regulation of innate immune responses in the brain. Nat Rev Immuno/ 9, 429439 (2009).

3. Diamond, B., Honig, G., Mader, S., Brimberg, L. \& Volpe, B.T. Brain-reactive antibodies and disease. Annu Rev Immunol 31, 345-385 (2013).

4. Deverman, B.E. \& Patterson, P.H. Cytokines and CNS development. Neuron 64, 61-78 (2009).

5. Petermann, F. \& Korn, T. Cytokines and effector T cell subsets causing autoimmune CNS disease. FEBS Lett 585, 3747-3757 (2011).

6. Cox, C.J., et al. Brain human monoclonal autoantibody from sydenham chorea targets dopaminergic neurons in transgenic mice and signals dopamine D2 receptor: implications in human disease. J Immunol 191, 5524-5541 (2013).

7. Brimberg, L., et al. Behavioral, pharmacological, and immunological abnormalities after streptococcal exposure: a novel rat model of Sydenham chorea and related neuropsychiatric disorders. Neuropsychopharmacology 37, 2076-2087 (2012).

8. Engelhardt, B. Development of the blood-brain barrier. Cell Tissue Res 314, 119-129 (2003).

9. Engelhardt, B. Neuroscience. Blood-brain barrier differentiation. Science 334, 1652-1653 (2011).

10. Jessen, N.A., Munk, A.S., Lundgaard, I. \& Nedergaard, M. The Glymphatic System: A Beginner's Guide. Neurochem Res (2015). 
11. Louveau, A., et al. Structural and functional features of central nervous system lymphatic vessels. Nature 523, 337-341 (2015).

12. Aspelund, A., et al. A dural lymphatic vascular system that drains brain interstitial fluid and macromolecules. J Exp Med 212, 991-999 (2015).

13. Bauer, H.C., et al. Neovascularization and the appearance of morphological characteristics of the blood-brain barrier in the embryonic mouse central nervous system. Brain Res Dev Brain Res 75, 269-278 (1993).

14. Ben-Zvi, A., et al. Mfsd2a is critical for the formation and function of the blood-brain barrier. Nature 509, 507-511 (2014).

15. Braniste, V., et al. The gut microbiota influences blood-brain barrier permeability in mice. Sci Transl Med 6, 263ra158 (2014).

16. Simister, N.E. Placental transport of immunoglobulin G. Vaccine 21, 3365-3369 (2003).

17. Banks, W.A., Kastin, A.J. \& Broadwell, R.D. Passage of cytokines across the blood-brain barrier. Neuroimmunomodulation 2, 241-248 (1995).

18. Tsao, N., Hsu, H.P., Wu, C.M., Liu, C.C. \& Lei, H.Y. Tumour necrosis factor-alpha causes an increase in blood-brain barrier permeability during sepsis. J Med Microbio/ 50, 812-821 (2001).

19. Ballabh, P., Braun, A. \& Nedergaard, M. The blood-brain barrier: an overview: structure, regulation, and clinical implications. Neurobiol Dis 16, 1-13 (2004).

20. Zhang, Y. \& Pardridge, W.M. Rapid transferrin efflux from brain to blood across the blood-brain barrier. J Neurochem 76, 1597-1600 (2001).

21. Roopenian, D.C. \& Akilesh, S. FcRn: the neonatal Fc receptor comes of age. Nat Rev Immunol 7, 715-725 (2007).

22. Montagne, A., et al. Blood-brain barrier breakdown in the aging human hippocampus. Neuron 85, 296-302 (2015).

23. Methia, N., et al. ApoE deficiency compromises the blood brain barrier especially after injury. Mol Med 7, 810-815 (2001).

24. Hafezi-Moghadam, A., Thomas, K.L. \& Wagner, D.D. ApoE deficiency leads to a progressive age-dependent blood-brain barrier leakage. Am J Physiol Cell Physiol 292, C1256-1262 (2007).

25. Hultman, K., Strickland, S. \& Norris, E.H. The APOE varepsilon4/varepsilon4 genotype potentiates vascular fibrin(ogen) deposition in amyloid-laden vessels in the brains of Alzheimer's disease patients. J Cereb Blood Flow Metab 33, 1251-1258 (2013).

26. Cerletti, A., Drewe, J., Fricker, G., Eberle, A.N. \& Huwyler, J. Endocytosis and transcytosis of an immunoliposome-based brain drug delivery system. J Drug Target 8, 435-446 (2000).

27. Kroll, S., et al. Control of the blood-brain barrier by glucocorticoids and the cells of the neurovascular unit. Ann N Y Acad Sci 1165, 228-239 (2009).

28. Gesuete, R., et al. Poly-ICLC preconditioning protects the blood-brain barrier against ischemic injury in vitro through type I interferon signaling. J Neurochem 123 Suppl 2, 7585 (2012).

29. Serres, S., et al. Magnetic resonance imaging reveals therapeutic effects of interferonbeta on cytokine-induced reactivation of rat model of multiple sclerosis. J Cereb Blood Flow Metab 33, 744-753 (2013).

30. Ni, C., et al. Interferon-gamma safeguards blood-brain barrier during experimental autoimmune encephalomyelitis. Am J Pathol 184, 3308-3320 (2014).

31. Veldhuis, W.B., et al. Interferon-beta prevents cytokine-induced neutrophil infiltration and attenuates blood-brain barrier disruption. J Cereb Blood Flow Metab 23, 1060-1069 (2003).

32. Niemela, J., et al. IFN-beta regulates CD73 and adenosine expression at the blood-brain barrier. Eur J Immunol 38, 2718-2726 (2008). 
33. Stone, L.A., et al. The effect of interferon-beta on blood-brain barrier disruptions demonstrated by contrast-enhanced magnetic resonance imaging in relapsing-remitting multiple sclerosis. Ann Neuro/ 37, 611-619 (1995).

34. Kowal, C., et al. Human lupus autoantibodies against NMDA receptors mediate cognitive impairment. Proc Natl Acad Sci U S A 103, 19854-19859 (2006).

35. Kowal, C., et al. Cognition and immunity; antibody impairs memory. Immunity 21, 179188 (2004).

36. Huerta, P.T., Kowal, C., DeGiorgio, L.A., Volpe, B.T. \& Diamond, B. Immunity and behavior: antibodies alter emotion. Proc Natl Acad Sci U S A 103, 678-683 (2006).

37. Mallard, C. Innate immune regulation by toll-like receptors in the brain. ISRN Neurol 2012, 701950 (2012).

38. Tuor, U.I., McKenzie, E. \& Tomanek, B. Functional magnetic resonance imaging of tonic pain and vasopressor effects in rats. Magn Reson Imaging 20, 707-712 (2002).

39. Abbott, N.J. Evidence for bulk flow of brain interstitial fluid: significance for physiology and pathology. Neurochem Int 45, 545-552 (2004).

40. Roth, J., Harre, E.M., Rummel, C., Gerstberger, R. \& Hubschle, T. Signaling the brain in systemic inflammation: role of sensory circumventricular organs. Front Biosci 9, 290-300 (2004).

41. Kabat, E.A., Glusman, M. \& Knaub, V. Quantitative estimation of the albumin and gamma globulin in normal and pathologic cerebrospinal fluid by immunochemical methods. Am J Med 4, 653-662 (1948).

42. Serafini, B., Rosicarelli, B., Magliozzi, R., Stigliano, E. \& Aloisi, F. Detection of ectopic Bcell follicles with germinal centers in the meninges of patients with secondary progressive multiple sclerosis. Brain Pathol 14, 164-174 (2004).

43. Solimena, M., et al. Autoantibodies to glutamic acid decarboxylase in a patient with stiffman syndrome, epilepsy, and type I diabetes mellitus. N Engl J Med 318, 1012-1020 (1988).

44. Alaedini, A., et al. Immune cross-reactivity in celiac disease: anti-gliadin antibodies bind to neuronal synapsin I. J Immunol 178, 6590-6595 (2007).

45. Gini, B., et al. Novel autoantigens recognized by CSF IgG from Hashimoto's encephalitis revealed by a proteomic approach. J Neuroimmunol 196, 153-158 (2008).

46. Nashi, E., Wang, Y. \& Diamond, B. The role of B cells in lupus pathogenesis. Int $J$ Biochem Cell Biol 42, 543-550 (2010).

47. Bradl, M., et al. Neuromyelitis optica: pathogenicity of patient immunoglobulin in vivo. Ann Neurol 66, 630-643 (2009).

48. Bravo-Zehnder, M., et al. Anti-ribosomal $P$ protein autoantibodies from patients with neuropsychiatric lupus impair memory in mice. Arthritis Rheumatol 67, 204-214 (2015).

49. Hahn, B.H. Antibodies to DNA. N Engl J Med 338, 1359-1368 (1998).

50. DeGiorgio, L.A., et al. A subset of lupus anti-DNA antibodies cross-reacts with the NR2 glutamate receptor in systemic lupus erythematosus. Nat Med 7, 1189-1193 (2001).

51. Gaynor, B., et al. Peptide inhibition of glomerular deposition of an anti-DNA antibody. Proc Natl Acad Sci U S A 94, 1955-1960 (1997).

52. Faust, T.W., et al. Neurotoxic lupus autoantibodies alter brain function through two distinct mechanisms. Proc Natl Acad Sci U S A 107, 18569-18574 (2010).

53. Vo, A., et al. Regional brain metabolism in a murine systemic lupus erythematosus model. J Cereb Blood Flow Metab 34, 1315-1320 (2014).

54. Mackay, M., et al. Brain metabolism and autoantibody titres predict functional impairment in systemic lupus erythematosus. Lupus Sci Med 2, e000074 (2015).

55. Chang, E.B.V., Meggan Mackay, Cynthia Aranow, Philip Watson, Czeslawa Kowal, Justin Storbeck, Paul Mattis, RoseAnn Berlin, Huiyi Chen, Simone Mader, Tomás S. Huerta, Patricio T. Huerta, Betty Diamond. Selective Impairment of Spatial Cognition 
Caused by Autoantibodies to the N-Methyl-d-Aspartate Receptor. E-BioMedicine 2, 753762 (2015).

56. Lapteva, L., et al. Anti-N-methyl-D-aspartate receptor antibodies, cognitive dysfunction, and depression in systemic lupus erythematosus. Arthritis Rheum 54, 2505-2514 (2006).

57. Omdal, R., et al. Neuropsychiatric disturbances in SLE are associated with antibodies against NMDA receptors. Eur J Neurol 12, 392-398 (2005).

58. Hanly, J.G., et al. Autoantibodies and neuropsychiatric events at the time of systemic lupus erythematosus diagnosis: results from an international inception cohort study.

Arthritis Rheum 58, 843-853 (2008).

59. Harrison, M.J., Ravdin, L.D. \& Lockshin, M.D. Relationship between serum NR2a antibodies and cognitive dysfunction in systemic lupus erythematosus. Arthritis Rheum 54, 2515-2522 (2006).

60. Husebye, E.S., et al. Autoantibodies to a NR2A peptide of the glutamate/NMDA receptor in sera of patients with systemic lupus erythematosus. Ann Rheum Dis 64, 1210-1213 (2005).

61. Kozora, E., et al. Antibodies against N-methyl-D-aspartate receptors in patients with systemic lupus erythematosus without major neuropsychiatric syndromes. J Neurol Sci 295, 87-91 (2010).

62. Petri, M., et al. Depression and cognitive impairment in newly diagnosed systemic lupus erythematosus. J Rheumatol 37, 2032-2038 (2010).

63. Arinuma, Y., Yanagida, T. \& Hirohata, S. Association of cerebrospinal fluid anti-NR2 glutamate receptor antibodies with diffuse neuropsychiatric systemic lupus erythematosus. Arthritis Rheum 58, 1130-1135 (2008).

64. Hirohata, S., Arinuma, Y., Yanagida, T. \& Yoshio, T. Blood-brain barrier damages and intrathecal synthesis of anti-N-methyl-D-aspartate receptor NR2 antibodies in diffuse psychiatric/neuropsychological syndromes in systemic lupus erythematosus. Arthritis Res Ther 16, R77 (2014).

65. Yoshio, T., Okamoto, H., Hirohata, S. \& Minota, S. IgG anti-NR2 glutamate receptor autoantibodies from patients with systemic lupus erythematosus activate endothelial cells. Arthritis Rheum 65, 457-463 (2013).

66. Matus, S., et al. Antiribosomal-P autoantibodies from psychiatric lupus target a novel neuronal surface protein causing calcium influx and apoptosis. J Exp Med 204, 32213234 (2007).

67. Elkon, K.B., Parnassa, A.P. \& Foster, C.L. Lupus autoantibodies target ribosomal P proteins. J Exp Med 162, 459-471 (1985).

68. Katzav, A., et al. Anti-P ribosomal antibodies induce defect in smell capability in a model of CNS -SLE (depression). J Autoimmun 31, 393-398 (2008).

69. Katzav, A., et al. Induction of autoimmune depression in mice by anti-ribosomal $P$ antibodies via the limbic system. Arthritis Rheum 56, 938-948 (2007).

70. Segovia-Miranda, F., et al. Pathogenicity of lupus anti-ribosomal $p$ antibodies: role of cross-reacting neuronal surface $p$ antigen in glutamatergic transmission and plasticity in a mouse model. Arthritis Rheumatol 67, 1598-1610 (2015).

71. Bonfa, E., et al. Association between lupus psychosis and anti-ribosomal P protein antibodies. N Engl J Med 317, 265-271 (1987).

72. Isshi, K. \& Hirohata, S. Association of anti-ribosomal P protein antibodies with neuropsychiatric systemic lupus erythematosus. Arthritis Rheum 39, 1483-1490 (1996).

73. Nagai, T., Yanagida, T. \& Hirohata, S. Anti-ribosomal P protein antibody induces Th1 responses by enhancing the production of IL-12 in activated monocytes. Mod Rheumatol 21, 57-62 (2011).

74. Madaio, M.P. Lupus autoantibodies 101: one size does not fit all; however, specificity influences pathogenicity. Clin Exp Immunol 131, 396-397 (2003). 
75. Hadjivassiliou, M., et al. The humoral response in the pathogenesis of gluten ataxia. Neurology 58, 1221-1226 (2002).

76. Lennon, V.A., et al. A serum autoantibody marker of neuromyelitis optica: distinction from multiple sclerosis. Lancet 364, 2106-2112 (2004).

77. Lennon, V.A., Kryzer, T.J., Pittock, S.J., Verkman, A.S. \& Hinson, S.R. IgG marker of optic-spinal multiple sclerosis binds to the aquaporin-4 water channel. J Exp Med 202, 473-477 (2005).

78. Roemer, S.F., et al. Pattern-specific loss of aquaporin-4 immunoreactivity distinguishes neuromyelitis optica from multiple sclerosis. Brain 130, 1194-1205 (2007).

79. Sorensen, A., Moffat, K., Thomson, C. \& Barnett, S.C. Astrocytes, but not olfactory ensheathing cells or Schwann cells, promote myelination of CNS axons in vitro. Glia 56, 750-763 (2008).

80. Marignier, R., et al. Oligodendrocytes are damaged by neuromyelitis optica immunoglobulin G via astrocyte injury. Brain 133, 2578-2591 (2010).

81. Palace, J., Leite, M.I., Nairne, A. \& Vincent, A. Interferon Beta treatment in neuromyelitis optica: increase in relapses and aquaporin 4 antibody titers. Arch Neurol 67, 1016-1017 (2010).

82. Wingerchuk, D.M., et al. International consensus diagnostic criteria for neuromyelitis optica spectrum disorders. Neurology (2015).

83. Wingerchuk, D.M., Lennon, V.A., Pittock, S.J., Lucchinetti, C.F. \& Weinshenker, B.G. Revised diagnostic criteria for neuromyelitis optica. Neurology 66, 1485-1489 (2006).

84. Waters, P., et al. Aquaporin-4 antibodies in neuromyelitis optica and longitudinally extensive transverse myelitis. Arch Neurol 65, 913-919 (2008).

85. Waters, P.J., et al. Serologic diagnosis of NMO: a multicenter comparison of aquaporin4-lgG assays. Neurology 78, 665-671; discussion 669 (2012).

86. Marignier, R., et al. Aquaporin-4 antibody-negative neuromyelitis optica: distinct assay sensitivity-dependent entity. Neurology 80, 2194-2200 (2013).

87. Takahashi, T., et al. Anti-aquaporin-4 antibody is involved in the pathogenesis of NMO: a study on antibody titre. Brain 130, 1235-1243 (2007).

88. Mader, S., et al. Patterns of antibody binding to aquaporin-4 isoforms in neuromyelitis optica. PLoS One 5, e10455 (2010).

89. Jarius, S. \& Wildemann, B. The history of neuromyelitis optica. J Neuroinflammation 10, 8 (2013).

90. Kim, H.J., et al. MRI characteristics of neuromyelitis optica spectrum disorder: an international update. Neurology 84, 1165-1173 (2015).

91. Saji, E., et al. Cognitive impairment and cortical degeneration in neuromyelitis optica. Ann Neurol 73, 65-76 (2013).

92. Nishiyama, S., et al. A case of NMO seropositive for aquaporin-4 antibody more than 10 years before onset. Neurology 72, 1960-1961 (2009).

93. Sabater, L., et al. Cytotoxic effect of neuromyelitis optica antibody (NMO-lgG) to astrocytes: an in vitro study. J Neuroimmunol 215, 31-35 (2009).

94. Pohl, M., et al. Pathogenic T cell responses against aquaporin 4. Acta Neuropathol 122, 21-34 (2011).

95. Mader, S., et al. Complement activating antibodies to myelin oligodendrocyte glycoprotein in neuromyelitis optica and related disorders. J Neuroinflammation 8, 184 (2011).

96. Bennett, J.L., et al. Intrathecal pathogenic anti-aquaporin-4 antibodies in early neuromyelitis optica. Ann Neurol 66, 617-629 (2009).

97. Saadoun, S., et al. Intra-cerebral injection of neuromyelitis optica immunoglobulin $\mathrm{G}$ and human complement produces neuromyelitis optica lesions in mice. Brain 133, 349-361 (2010). 
98. Misu, T., et al. Presence of six different lesion types suggests diverse mechanisms of tissue injury in neuromyelitis optica. Acta Neuropathol 125, 815-827 (2013).

99. Kowarik, M.C., et al. The cerebrospinal fluid immunoglobulin transcriptome and proteome in neuromyelitis optica reveals central nervous system-specific B cell populations. J Neuroinflammation 12, 19 (2015).

100. Chihara, N., et al. Plasmablasts as migratory IgG-producing cells in the pathogenesis of neuromyelitis optica. PLoS One 8, e83036 (2013).

101. Pohl, M., et al. T cell-activation in neuromyelitis optica lesions plays a role in their formation. Acta Neuropathol Commun 1, 85 (2013).

102. Jones, M.V., Huang, H., Calabresi, P.A. \& Levy, M. Pathogenic aquaporin-4 reactive T cells are sufficient to induce mouse model of neuromyelitis optica. Acta Neuropathol Commun 3, 28 (2015).

103. Kinoshita, M., et al. Neuromyelitis optica: Passive transfer to rats by human immunoglobulin. Biochem Biophys Res Commun 386, 623-627 (2009).

104. Kitic, M., et al. Intrastriatal injection of interleukin-1 beta triggers the formation of neuromyelitis optica-like lesions in NMO-IgG seropositive rats. Acta Neuropathol Commun 1, 5 (2013).

105. Bradl, M. \& Lassmann, H. Experimental models of neuromyelitis optica. Brain Pathol 24, 74-82 (2014).

106. Palmeira, P., Quinello, C., Silveira-Lessa, A.L., Zago, C.A. \& Carneiro-Sampaio, M. IgG placental transfer in healthy and pathological pregnancies. Clin Dev Immunol 2012, 985646 (2012).

107. Singalavanija, S., Limpongsanurak, W. \& Aoongern, S. Neonatal lupus erythematosus: a 20-year retrospective study. J Med Assoc Thai 97 Suppl 6, S74-82 (2014).

108. Hacohen, Y., et al. Fetal acetylcholine receptor inactivation syndrome: A myopathy due to maternal antibodies. Neurol Neuroimmunol Neuroinflamm 2, e57 (2015).

109. Vinet, E., et al. Neurodevelopmental disorders in children born to mothers with systemic lupus erythematosus. Lupus 23, 1099-1104 (2014).

110. Lahita, R.G. Systemic lupus erythematosus: learning disability in the male offspring of female patients and relationship to laterality. Psychoneuroendocrinology 13, 385-396 (1988).

111. McAllister, D.L., et al. The influence of systemic lupus erythematosus on fetal development: cognitive, behavioral, and health trends. J Int Neuropsychol Soc 3, 370376 (1997).

112. Ross, G., Sammaritano, L., Nass, R. \& Lockshin, M. Effects of mothers' autoimmune disease during pregnancy on learning disabilities and hand preference in their children. Arch Pediatr Adolesc Med 157, 397-402 (2003).

113. Urowitz, M.B., et al. Neurocognitive abnormalities in offspring of mothers with systemic lupus erythematosus. Lupus 17, 555-560 (2008).

114. Comi, A.M., Zimmerman, A.W., Frye, V.H., Law, P.A. \& Peeden, J.N. Familial clustering of autoimmune disorders and evaluation of medical risk factors in autism. $J$ Child Neurol 14, 388-394 (1999).

115. Lee, J.Y., et al. Neurotoxic autoantibodies mediate congenital cortical impairment of offspring in maternal lupus. Nat Med 15, 91-96 (2009).

116. Wang, L., et al. Female mouse fetal loss mediated by maternal autoantibody. J Exp Med 209, 1083-1089 (2012).

117. Vinet, E., et al. Increased male-to-female ratio among children born to women with systemic lupus erythematosus: comment on the article by Lockshin et al. Arthritis Rheum 65, 1129 (2013).

118. Jagota, P., Vincent, A. \& Bhidayasiri, R. Transplacental transfer of NMDA receptor antibodies in an infant with cortical dysplasia. Neurology 82, 1662-1663 (2014). 
119. Brimberg, L., Sadiq, A., Gregersen, P.K. \& Diamond, B. Brain-reactive IgG correlates with autoimmunity in mothers of a child with an autism spectrum disorder. Mol Psychiatry 18, 1171-1177 (2013).

120. Braunschweig, D., et al. Autism-specific maternal autoantibodies recognize critical proteins in developing brain. Transl Psychiatry 3, e277 (2013).

121. Croen, L.A., et al. Maternal mid-pregnancy autoantibodies to fetal brain protein: the early markers for autism study. Biol Psychiatry 64, 583-588 (2008).

122. Zimmerman, A.W., et al. Maternal antibrain antibodies in autism. Brain Behav Immun 21, 351-357 (2007).

123. Singer, H.S., et al. Prenatal exposure to antibodies from mothers of children with autism produces neurobehavioral alterations: A pregnant dam mouse model. J Neuroimmunol 211, 39-48 (2009).

124. Bauman, M.D., et al. Maternal antibodies from mothers of children with autism alter brain growth and social behavior development in the rhesus monkey. Transl Psychiatry 3, e278 (2013).

125. Dalton, P., et al. Maternal neuronal antibodies associated with autism and a language disorder. Ann Neurol 53, 533-537 (2003).

126. Martin, L.A., et al. Stereotypies and hyperactivity in rhesus monkeys exposed to IgG from mothers of children with autism. Brain Behav Immun 22, 806-816 (2008).

127. Braunschweig, D., et al. Maternal autism-associated IgG antibodies delay development and produce anxiety in a mouse gestational transfer model. J Neuroimmunol 252, 56-65 (2012).

128. Camacho, J., et al. Embryonic intraventricular exposure to autism-specific maternal autoantibodies produces alterations in autistic-like stereotypical behaviors in offspring mice. Behav Brain Res 266, 46-51 (2014).

129. Martinez-Cerdeno, V., et al. Prenatal Exposure to Autism-Specific Maternal Autoantibodies Alters Proliferation of Cortical Neural Precursor Cells, Enlarges Brain, and Increases Neuronal Size in Adult Animals. Cereb Cortex (2014).

130. Atladottir, H.O., et al. Association of family history of autoimmune diseases and autism spectrum disorders. Pediatrics 124, 687-694 (2009).

131. Braunschweig, D., et al. Behavioral correlates of maternal antibody status among children with autism. J Autism Dev Disord 42, 1435-1445 (2012).

132. Saadoun, S., et al. Neuromyelitis optica IgG causes placental inflammation and fetal death. J Immunol 191, 2999-3005 (2013).

133. Igel, C., et al. Neuromyelitis optica in pregnancy complicated by posterior reversible encephalopathy syndrome, eclampsia and fetal death. J Clin Med Res 7, 193-195 (2015).

134. Reuss, R., et al. A woman with acute myelopathy in pregnancy: case outcome. BMJ 339, b4026 (2009).

135. Bloom, O., et al. Generation of a unique small molecule peptidomimetic that neutralizes lupus autoantibody activity. Proc Natl Acad Sci U S A 108, 10255-10259 (2011).

136. Shams'ili, S., et al. Paraneoplastic cerebellar degeneration associated with antineuronal antibodies: analysis of 50 patients. Brain 126, 1409-1418 (2003).

137. Graus, F., et al. Anti-Hu antibodies in patients with small-cell lung cancer: association with complete response to therapy and improved survival. J Clin Oncol 15, 2866-2872 (1997).

138. Dogan Onugoren, M., et al. Limbic encephalitis due to GABAB and AMPA receptor antibodies: a case series. J Neurol Neurosurg Psychiatry 86, 965-972 (2015).

139. Gozzard, P., et al. Paraneoplastic neurologic disorders in small cell lung carcinoma: A prospective study. Neurology 85, 235-239 (2015). 
140. Orange, D., et al. Cellular immune suppression in paraneoplastic neurologic syndromes targeting intracellular antigens. Arch Neurol 69, 1132-1140 (2012).

141. Lai, M., et al. AMPA receptor antibodies in limbic encephalitis alter synaptic receptor location. Ann Neurol 65, 424-434 (2009).

142. Saiz, A., et al. Spectrum of neurological syndromes associated with glutamic acid decarboxylase antibodies: diagnostic clues for this association. Brain 131, 2553-2563 (2008).

143. Dalmau, J., Lancaster, E., Martinez-Hernandez, E., Rosenfeld, M.R. \& Balice-Gordon, R. Clinical experience and laboratory investigations in patients with anti-NMDAR encephalitis. Lancet Neurol 10, 63-74 (2011).

144. Irani, S.R., et al. Antibodies to Kv1 potassium channel-complex proteins leucine-rich, glioma inactivated 1 protein and contactin-associated protein-2 in limbic encephalitis, Morvan's syndrome and acquired neuromyotonia. Brain 133, 2734-2748 (2010).

145. Murinson, B.B. \& Guarnaccia, J.B. Stiff-person syndrome with amphiphysin antibodies: distinctive features of a rare disease. Neurology 71, 1955-1958 (2008).

146. Kastrup, O., Meyring, S. \& Diener, H.C. Atypical paraneoplastic brainstem encephalitis associated with anti-ri-antibodies due to thymic carcinoma with possible clinical response to immunoglobulins. Eur Neurol 45, 285-287 (2001).

147. Graus, F., et al. Anti-Hu-associated paraneoplastic encephalomyelitis: analysis of 200 patients. Brain 124, 1138-1148 (2001).

148. Monstad, S.E., et al. Yo antibodies in ovarian and breast cancer patients detected by a sensitive immunoprecipitation technique. Clin Exp Immunol 144, 53-58 (2006).

149. Lukacs, S., Szabo, N. \& Woodhams, S. Rare association of anti-hu antibody positive paraneoplastic neurological syndrome and transitional cell bladder carcinoma. Case Rep Urol 2012, 724940 (2012).

150. Yamamoto, T. \& Tsuji, S. [Anti-Ma2-associated encephalitis and paraneoplastic limbic encephalitis]. Brain Nerve 62, 838-851 (2010).

151. Kirvan, C.A., Swedo, S.E., Heuser, J.S. \& Cunningham, M.W. Mimicry and autoantibodymediated neuronal cell signaling in Sydenham chorea. Nat Med 9, 914-920 (2003).

152. O'Connor, K.C., et al. Self-antigen tetramers discriminate between myelin autoantibodies to native or denatured protein. Nat Med 13, 211-217 (2007).

153. Pleasure, D. Diagnostic and pathogenic significance of glutamate receptor autoantibodies. Arch Neurol 65, 589-592 (2008).

154. Hughes, E.G., et al. Cellular and synaptic mechanisms of anti-NMDA receptor encephalitis. J Neurosci 30, 5866-5875 (2010).

155. Cohen-Kashi Malina, K., Ganor, Y., Levite, M. \& Teichberg, V.I. Autoantibodies against an extracellular peptide of the GluR3 subtype of AMPA receptors activate both homomeric and heteromeric AMPA receptor channels. Neurochem Res 31, 1181-1190 (2006).

156. Dalakas, M.C., Fujii, M., Li, M. \& McElroy, B. The clinical spectrum of anti-GAD antibodypositive patients with stiff-person syndrome. Neurology 55, 1531-1535 (2000).

157. Geis, C., et al. Stiff person syndrome-associated autoantibodies to amphiphysin mediate reduced GABAergic inhibition. Brain 133, 3166-3180 (2010).

158. Hadjivassiliou, M., et al. Autoantibody targeting of brain and intestinal transglutaminase in gluten ataxia. Neurology 66, 373-377 (2006).

159. Lai, M., et al. Investigation of LGl1 as the antigen in limbic encephalitis previously attributed to potassium channels: a case series. Lancet Neurol 9, 776-785 (2010).

160. Lancaster, E., et al. Investigations of caspr2, an autoantigen of encephalitis and neuromyotonia. Ann Neurol 69, 303-311 (2011).

161. Schiess, N. \& Pardo, C.A. Hashimoto's encephalopathy. Ann N Y Acad Sci 1142, 254265 (2008). 


\section{Figure Legend}

Figure 1: Mechanism of action of autoantibodies penetrating brain tissue. Autoantibodies that bind to cell surface proteins on glial cells or neuronal cells can induce complement mediated cytotoxicity (CDC), which results in the formation of the membrane attack complex (MAC) (1). In contrast, antibody-dependent cell-mediated cytotoxicity (ADCC) is induced by Fc receptor bearing effector cells that can lyse antibody coated target cells (2) CDC and ADCC can result in cell death. Antibodies can interfere with the crosstalk of receptor signalling by acting as agonistic, antagonistic or co-agonistic antibodies (3). Antibodies can cause receptor internalization following binding of their cell surface antigen, which leads to altered antigen density on the cell surface (4). Some brain reactive antibodies will have no functional effect (5). 


\section{Outstanding Questions}

- What mechanisms regulate the integrity of the blood-brain barrier?

- What approaches can be used to assess barrier integrity in a non-invasive manner?

- Are there other autoimmune diseases that exhibit antibodies to neuronal antigens? Are there neuronal pathologies or behavioral abnormalities associated with these diseases?

- Are there other infectious diseases where brain pathology and behavioral disorders can be associated with autoantibodies?

- What renders a brain susceptible to antibody-mediated injury? Under circumstances where barrier integrity is broken, some individuals do not exhibit brain pathology. What features of the tissue microenvironment and immune cell types determine a pathogenic response? What features of the autoantibodies determine a pathogenic response?

- Why do some antibodies appear to selectively target cells in the CNS when they are response to antigen that is expressed also in peripheral tissue? 


\section{Figure}

1. Complement

activation
2. Antibody mediated

cell cytotoxicity
3. Agonistic/Antagonistic/

Co-agonistic antibody
4. Receptor

internalization
5. no functional effect
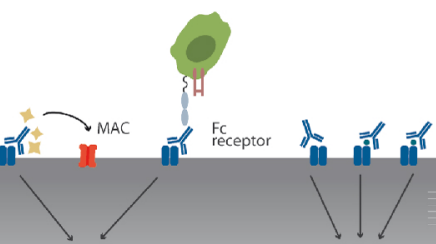

cell death

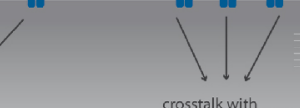

crosstalk with
receptor signalling

Altered antigen
density on cell surface

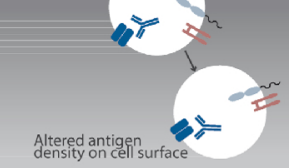
Blood Brain Barrier

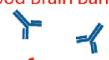

Pericyte

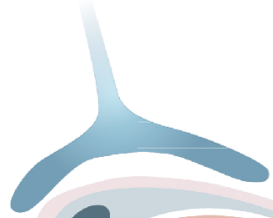

Antibody penetration across the
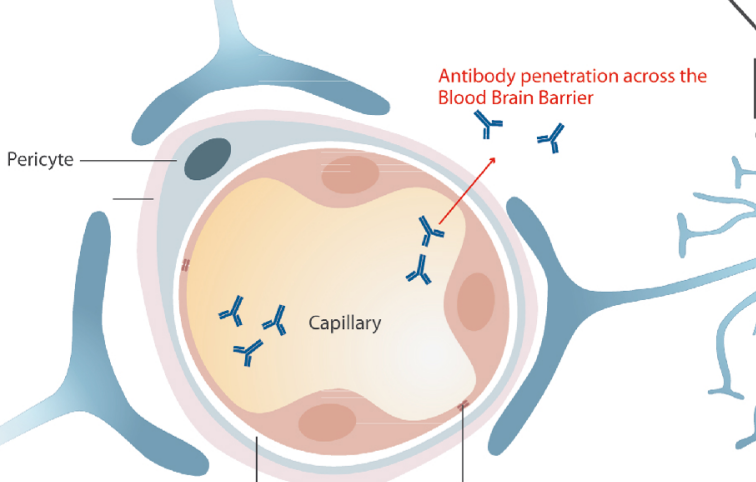

\& \& Capillary ii
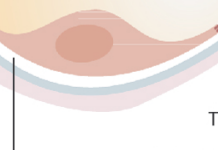

Tight junction

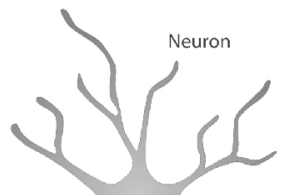

Basement Endothelial cell membrane 